\title{
THE GoOd, THE BAD AND THE WinNer: BUENOS POLÍTICOS Y ÉXITO ELECTORAL eN AmÉRICA LATINA*
}

\author{
The Good, the Bad and the Winner: \\ Good Politicians and Electoral Success in Latin America
}

MARÍA DEL MAR MARTÍNEZ ROSÓN

Universidad Autónoma de Barcelona

\begin{abstract}
RESUMEN
El objetivo de este trabajo es analizar las cualidades que definen a un buen político desde la propia perspectiva de los legisladores latinoamericanos. Además de comprobar si estas coinciden o no con la valoración de los ciudadanos, también se realiza una comparación con las cualidades más relevantes para ganar elecciones. Asimismo, distintos análisis han identificado que el grado de conocimientos políticos altera estas preferencias, haciendo el carisma más atractivo entre los ciudadanos políticamente menos sofisticados. Sin embargo, poco se sabe del impacto de esta variable entre los políticos. Se trata de comprobar, por tanto, a) si la formación y los conocimientos políticos también alteran la percepción de los diputados sobre qué características son más valoradas y b) cuáles sirven mejor para ganar elecciones, así como c) analizar la influencia de la sofisticación política de su electorado.
\end{abstract}

Palabras clave: Cualidades personales, carisma, capacidad, conocimientos políticos, América Latina.

\begin{abstract}
In this paper I analyze what personal traits define a good politician from the perspective of the Latin-American legislators. In addition to verifying whether these qualities are the same elected by citizens, in this work I compare them with the qualities that serve to win elections. Furthermore, different analyses remark the effect that political knowledge has on these preferences, making charisma more attractive among citizens with low levels of political sophistication. However, little is known about the impact of this variable for politicians. This article measures a)whether education and political knowledge also alter the perception of the deputies on what characteristics are more valued $b$ ) which of these characteristics serve better to gain elections, as well as c) the influence of constituents' political sophistication.
\end{abstract}

Key words: Personal traits, charisma, competence, political knowledge, Latin America.

* Los datos que se analizan en este artículo proceden del proyecto dirigido por Manuel Alcántara “Congruencia Política y Representación: Elite Parlamentaria y Opinión Pública en América Latina” CSO2012-39377-C02-01 del Ministerio de Economía y Competitividad, así como de los proyectos previos obtenidos desde 1994 y del que la autora es miembro. Agradezco los comentarios y sugerencias realizados por los dos revisores anónimos de la revista que sin duda han ayudado a mejorar y enriquecer el documento final. 


\section{INTRODUCCIÓN}

En la literatura politológica centrada en las democracias latinoamericanas se ha investigado sobre los procesos de democratización y de consolidación, las relaciones entre sus legislativos y ejecutivos, sus sistemas de partidos y electorales o los movimientos sociales y revolucionarios acontecidos, entre otros. Más recientemente se ha empezado a estudiar la cercanía o lejanía entre ciudadanos y políticos en sus posiciones ideológicas y sus preferencias en distintos issues de política pública para identificar el grado de congruencia entre ambos. Llama la atención, sin embargo, que apenas se haya prestado atención a las características y cualidades de los políticos o de los candidatos. Si bien es importante conocer el grado de solapamiento entre la opinión de representantes y representados en la escala ideológica izquierda-derecha, también es relevante conocer el grado de solapamiento en los aspectos no ideológicos o como Stokes (1963) denomina, en los valence issues. Los aspectos personales y las cualidades individuales de los políticos tienen más importancia de la que generalmente le otorgan los investigadores. Y esto es aun más evidente en aquellos países donde el grado de institucionalización de los sistemas de partidos es bajo. Según Mainwaring y Torcal (2005), los sistemas de partidos que se desarrollan en las democracias menos avanzadas tienen una mayor volatilidad electoral y la conexión entre los partidos y los ciudadanos tiene un menor componente ideológico y programático. Además, el vínculo entre los votantes y los candidatos está construido sobre aspectos relacionados con las características personales de estos últimos en vez de apoyarse en aspectos partidistas como la ideología o el programa. Si esta descripción, que detallaría perfectamente el panorama político de diversos países latinoamericanos, es ampliamente aceptada por los investigadores, ¿por qué no hemos prestado atención a las cualidades de los políticos? ¿Qué cualidades tienen en común los políticos bien valorados? ¿Son estas características personales las mismas que influyen en el voto de los ciudadanos?

Los trabajos publicados hasta el momento muestran que las características personales de los candidatos importan no solo por la valoración que los ciudadanos pueden hacer de ellas, sino porque influyen en el apoyo a los partidos (Abney et al., 2013), la decisión del voto (Kulisheck y Mondak, 1996; Prysby, 2008; Welch y Hibbing, 1997) tiene implicaciones en las posibilidades de reelección (Abramowitz, 1991; Finan y Ferraz, 2005; Stone et al. 2004), en la selección de candidatos (Galasso y Nannicini, 2011), en los resultados de elecciones primarias (Mondak, 1995; Welch y Hibbing, 1997), en la campaña electoral (Hayes, 2005; Funk, 1997 y 1999) o en las posiciones ideológicas de los partidos (Stone y Simas, 2010). A pesar de estos hallazgos, el tema ha sido relegado a un segundo plano en la literatura, pero los investigadores subrayan que la evaluación de estas cualidades no debe ser juzgada como una actividad superflua ${ }^{1}$, sino que debe ser entendida como

\footnotetext{
Por ejemplo, en el modelo espacial de Downs se considera el voto no ideológico como un voto irracional. En la crítica de Stokes (1963), a los modelos espaciales este autor añade los valence issues como todas aquellas cuestiones que los ciudadanos vinculan con el partido o el candidato, que no encajan en el modelo espacial, y a los que se le otorga un valor positivo o negativo. Otros autores posteriormente han acotado el concepto de valencia incluyendo solo aquellas características que muestran el carácter y las habilidades de los políticos
} 
una forma racional de toma de decisiones (Page, 1978; Fiorina, 1981). Ante situaciones de información incompleta y debido a los costes de adquirirla, las cualidades personales funcionan como atajo informativo, igual que podrían acudir a la ideología o al partido. ${ }^{2}$

Las investigaciones no solo han planteado la racionalidad del uso de las cualidades personales, además, han demostrado que la observación se realiza de forma espontánea (McCurly y Mondak, 1995; Popkin, 1991; Sullivan et al., 1990), ya que hay una tendencia natural a considerar esta información y por tanto no es necesaria una socialización previa (Canache et al., 2000). También hay que resaltar que la inclusión de las características personales en los procesos de valoración y en otras decisiones como el voto, no está subyugada a la ausencia de otra información como podría ser el partido político al que pertenece el candidato o su posición en distintos temas de política pública. ${ }^{3}$ Independientemente de que exista otra información y de la cantidad de información personal que esté disponible, esta será tenida en cuenta por los electores (Kulisheck y Mondak, 1996). En definitiva, sería irracional que los votantes no dejaran fuera a aquellos que son considerados como malos políticos y un sistema electoral donde la calidad importa y se comporta bien con la esencia de la democracia representativa (Kulisheck y Mondak, 1996).

Los estudios desarrollados en otros espacios regionales han llegado a la conclusión de que la honradez y la competencia son las dos cualidades con más peso en las valoraciones de los candidatos realizadas por los ciudadanos. Sin embargo, otras variables como el grado de conocimientos políticos alteran estas preferencias, de forma que aquellos ciudadanos políticamente menos sofisticados responden mejor que los más sofisticados a otras características como el carisma. Aunque esto se ha comprobado en el ámbito de los electores poco se sabe de cómo perciben y actúan los políticos ante este hecho. El análisis de los datos procedentes de los diputados latinoamericanos va a hacer posible comprobar si el nivel de formación y los conocimientos políticos también alteran la percepción que tienen sobre qué características son mejores, si por el contrario es el grado de conocimientos políticos de su electorado el que modifica sus preferencias, o si ambas son ciertas.

Para abordar esta cuestión se han utilizado los datos de opinión de los diputados latinoamericanos procedentes del Proyecto Elites Parlamentarias Latinoamericanas

(Stone y Simas, 2010). Un buen número de trabajos ha usado el concepto de valencia como equivalente a competencia (Clark, 2009 y Groseclose, 2001) o calidad (Galasso y Nannicini, 2011).

2 Por ejemplo, Lau y Redlawsk (2001) agrupan en cinco categorías los distintos atajos o heurísticos usados por los votantes: afiliación partidista, ideología política, grupos de apoyo, la viabilidad del candidato según las encuestas y la apariencia del candidato. También se puede consultar Huckfeldt et al. (2005).

3 Algunos autores han encontrado que las características de competencia influyen también en contextos donde el partido es más importante que el candidato. Así, por ejemplo, Green (2007) destaca que en la política británica se ha producido un aumento de la competición entre partidos basada en la calidad de los candidatos como resultado de una convergencia ideológica entre la izquierda y la derecha. También se puede consultar Clark (2009) para nueve democracias europeas y Galasso y Nannicini (2011) para el caso italiano. Sobre el impacto que tienen los distintos diseños electorales en el voto personal puede consultarse Shugart, Valdini y Suominen (2005). 
(PELA) de la Universidad de Salamanca. ${ }^{4}$ Este proyecto recaba información en los parlamentos desde 1994 de forma individual y personal entrevistando a una muestra representativa de diputados de cada país en cada período legislativo. Los cuestionarios incluyen preguntas muy diversas sobre democracia, partidos, ideología, funciones del Estado, problemas del país, valores y religión, entre otros. Los análisis de este trabajo se han realizado a partir de los cuestionarios de 1.379 diputados de las cámaras legislativas de 10 países latinoamericanos en 18 períodos legislativos (ver Anexo I).

El artículo se organiza de la siguiente forma. En primer lugar se ofrecen algunas precisiones sobre el estudio de las cualidades y características de los políticos en la literatura y las dimensiones en que se agrupan. En un segundo momento se describen las características asociadas con un buen político según los diputados latinoamericanos y las características vinculadas al éxito electoral. En tercer lugar se realiza un análisis multinomial con el fin de ver cómo influye la sofisticación política de los diputados y de su electorado en la definición que tienen de un buen político y de un político ganador. El artículo finaliza con el compendio de los hallazgos más interesantes.

\section{CUALIDADES PERSONALES DE LOS POLÍTICOS}

Antes de abordar qué características son las que definen a un buen político hay que determinar cómo organizar y priorizar esas características. Esta decisión metodológica ofrece resultados muy distintos y este trabajo se fundamenta en estas diferencias. Es posible encontrar dos formas de abordar el estudio de las cualidades políticas en la literatura. La diferencia radica en cómo se realiza la medición de estas cualidades, una usando un procedimiento normativo y, otra, que se podría denominar utilitaria. La primera opción y la más extendida en la literatura declara un conjunto de características que serían deseables en los individuos. A este conjunto de características se le otorgan ciertas puntuaciones, iguales o no, la suma de las cuales define el nivel de calidad ${ }^{5}$. La segunda de las opciones tiene un sentido más práctico, ya que no se basa en una estructura rígida sino que se construye a partir de un conglomerado flexible de características. Esta opción, menos desarrollada y utilizada, consistiría en entender como elementos de calidad aquellos que otorgan a los candidatos el éxito electoral. ${ }^{6}$ De este modo, las dimensiones que finalmente dibujan a un buen político no solo serían diferentes entre países, sino también entre regiones o partidos, en función de las características que hayan tenido más peso durante la elección. El éxito electoral sería para esta opción la variable a partir de la cual se pueden identificar las características importantes. En la primera opción el éxito electoral no es un parteaguas ni condición sine qua non para que determinada característica sea mejor valorada, en la segunda sí.

\footnotetext{
4 Alcántara (Director), 1994-2016. Se puede consultar más información sobre el proyecto, acceder a las bases de datos y los cuestionarios en la siguiente dirección: http://americo.usal.es/oir/elites/bases_de_datos.htm Véanse Squire (1993 y 1998), Squire y Hamm (2005), Stone et al. (2004), Moncrief et al. (2001) y Hall y Bonneau (2006).

Véase Kinder et al. (1980).
} 
Este trabajo se construye a partir de estas dos posibilidades y se centra en el análisis de las implicaciones que acarrean cada una de ellas. Las diferencias entre ambas formas de medir y definir a un buen político, una de carácter normativo y otra de carácter práctico, tiene efectos importantes. Dar por buenas las cualidades que otorgan más votos puede conducir a afirmar que comportamientos corruptos o clientelares, por ejemplo, son características deseables. Por el contrario, manteniendo una estructura fija de las cualidades que se entienden por buenas puede verse hasta qué punto estas influyen sobre las valoraciones o el voto, y comparar estas con otras variables clásicas de explicación del voto como pueden ser la ideología, el partido o la posición en distintos ámbitos de políticas públicas. El objetivo de este artículo es establecer un diálogo entre ambas concepciones comparando el conjunto concreto de características que ellos mismos consideran como deseables en un buen político con las cualidades que son necesarias para resultar elegido. Esta comparación permite ver qué características son necesarias para cumplir con las funciones de un representante frente a las cualidades necesarias para ganar una elección. Además, permite identificar qué valores deben subrayar los candidatos en una campaña electoral, cuáles ocultar, así como qué características del candidato opositor deben atacar y cuáles resaltar. Además, el análisis permite comprobar el grado de congruencia entre ciudadanos y políticos en aspectos no ideológicos, cuestión primordial en el tema de la representación (Miller y Stokes, 1963).

Por tanto, este trabajo pretende responder a dos preguntas. La primera cuestión es identificar las diferencias entre las características mejor valoradas en un buen político y aquellas que convierten a un político en un ganador de la contienda electoral. ${ }^{7} \mathrm{El}$ segundo interrogante aborda el impacto de la sofisticación política tanto del político como del elector (entendida como una suma entre educación y conocimientos políticos) sobre las cualidades más valoradas en un buen político y sobre las características más importantes que intervienen en el éxito electoral.

Pero ¿cuáles son las cualidades que definen a un buen político? Las características recogidas en la literatura son muy variadas y oscilan desde concepciones muy simplistas hasta otras que recogen un sinfín de adjetivos. En la actualidad lo habitual es que se organicen las distintas cualidades bajo cuatro dimensiones amplias: ${ }^{8}$ competencia, liderazgo, integridad y carisma (Kinder, 1983). La aplicación a situaciones prácticas ha servido para identificar el peso que tiene cada uno de estos criterios y es por ello que distintos trabajos han puesto el acento únicamente en dos características: la integridad y

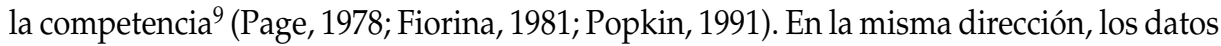
empíricos apuntan a que tanto la competencia como la integridad tienen un impacto más estable y significativo. Las evaluaciones de los ciudadanos parecen responder de forma clara y semejante, en distintos contextos, a los elementos de competencia e integridad, mientras que el liderazgo y el carisma cumplen una función menos clara (Funk, 1999).

7 Sobre la carrera política y el impacto de la ambición en el comportamiento político para tres casos centroamericanos puede consultarse Martínez Rosón (2011).

8 Una aplicación reciente se encuentra en Prysby (2008).

9 Véase Funk (1999), McCurley y Mondak (1995) y Mondak (1995). Kinder et al. (1980) estudian todas aquellas características que los ciudadanos valoran positiva o negativamente de los candidatos. 
A pesar de todo, esta autora considera que no debe abandonarse la atención sobre el liderazgo y el carisma, en virtud de la importancia que los propios políticos otorgan a ambos aspectos. ¿Es cierta esta importancia entre los políticos latinoamericanos? Y una cuestión aun más relevante, ipor qué le dan esta importancia?

El carisma dota a quien lo posee de capacidades especiales que fascinan al observador. Como Merolla y Zechmeister destacan, que los ciudadanos vean a un político como un líder carismático tiene ciertas ventajas. Fundamentalmente, a los líderes carismáticos se les ofrece una protección (o efecto teflón) frente a las consecuencias que tendría un mal rendimiento político. El carisma protege la imagen del político evitando que los individuos le culpen de los malos resultados (2011: 30). No es de extrañar, por tanto, que cualquier político encuentre que el carisma es un factor importante si está asociado a capacidades especiales $y$, si además, garantiza cierta benevolencia ciudadana frente a los errores políticos que se puedan cometer.

Otra posible razón podría estar en el impacto que el carisma parece tener entre las audiencias poco informadas. Los experimentos de Funk $(1996$; 1997) ponen de relieve que los ciudadanos en general aprecian la competencia por encima del carisma. Sin embargo, cuando se diferencia entre ciudadanos más o menos informados, estos últimos, si bien responden positivamente a la competencia, no parecen preferirla sobre el carisma. ${ }^{10}$ Las implicaciones son claras: las audiencias menos informadas son más vulnerables a la información ofrecida por los medios y a los contenidos de las campañas electorales. Si esta conclusión tuviera una aplicación generalizable sería especialmente importante para el contexto latinoamericano, ya que el porcentaje de población en esta situación es mayor que en las viejas democracias.

En un sentido similar Zaller (1992) defiende que las opiniones de los ciudadanos están modeladas por el discurso de la élite que ofrecen los medios de comunicación. Sin embargo, al igual que Funk, cree que el impacto de los discursos depende del grado de conocimientos e interés del receptor. Los ciudadanos con mayor interés y con más conocimientos políticos reciben más información, ya que están más expuestos, pero a su vez son más reacios a aceptar todo aquello que sea inconsistente con sus valores. Por el contrario, los ciudadanos con menos conocimientos, aunque reciben menos información, son menos eficaces en rechazar todo aquello que sea ideológicamente inconsistente con sus valores.

La literatura centrada en atajos informativos y heurísticos también ha analizado el impacto de la formación y del nivel de sofisticación política de los votantes como elemento clave en el uso de esos atajos. El trabajo de Lau y Redlawsk (2001) muestra que los ciudadanos usan estos atajos informativos para tomar decisiones políticas y su uso aumenta cuando el acceso a la información es más difícil. Sin embargo, los ciudadanos políticamente poco sofisticados usan atajos distintos. Mientras que los ciudadanos sofisticados se centran más

10 El impacto del grado de sofisticación política de los ciudadanos así como del grado de formación ha sido también destacado por otros estudios: Lau y Redlawsk (2008), Gordon y Segura (1997), Krassa (1990), Kuklinski et al. (2001), Kuklinski y Quirk (2001), Fisher et al. (2008), Jerit et al. (2006). 
en la información sobre la ideología y los grupos de apoyo del político, los ciudadanos políticamente menos sofisticados se apoyan en la apariencia y la información del partido al que pertenece. Además, los votantes complejos usan mejor los atajos, emplean la poca información que tienen mejor que los votantes no complejos. En definitiva, el trabajo de estos autores pone de manifiesto que el uso de la información sobre el candidato varía en función del grado de sofisticación política del ciudadano.

Si bien entre los ciudadanos el bajo nivel educativo y la falta de conocimientos políticos pueden explicar la importancia del carisma, en el ámbito de los diputados el análisis de este aspecto es un poco más complejo. La explicación por la que algunos diputados dan más importancia al carisma o al liderazgo puede estar influenciada por el nivel de sofisticación propio (medida como el nivel de educación y de conocimientos políticos, como defienden Zaller y Funk), por el nivel de su electorado (como se puede derivar de Miller y Stokes), o por ambos. Un diputado con menores conocimientos políticos, con menor educación o con menor experiencia puede, igual que los ciudadanos, no dar más importancia a la honradez que al carisma. Al igual que Funk proponía en su estudio, (hipótesis 1) si los ciudadanos con menos conocimientos no prefieran la competencia frente al carisma los diputados no deberían escapar a esa relación y por tanto cabe esperar que los diputados con menor nivel educativo y sofisticación política tengan las mismas preferencias por la honradez, la capacidad y el carisma. Por el contrario, si la tesis de Funk no se cumple entonces es más probable que tanto los diputados con más conocimientos políticos como los que no, preferirán la honradez y la capacidad por encima del carisma.

También puede suceder que a la hora de decidir qué características definen a un buen político, los encuestados se estén dejando guiar en su respuesta por las preferencias de su electorado. En la relación entre los representantes y los representados la forma en la que se produce la congruencia entre ambos es un tema importante. Miller y Stokes (1963: 50) establecen dos métodos distintos por los cuales los ciudadanos pueden influir sobre sus diputados. ${ }^{11}$ En primer lugar puede suceder que realmente los votantes elijan a un representante con ideas congruentes con las suyas. En una segunda vía la congruencia se produce porque el diputado sigue lo que él piensa que su distrito quiere (bien para lograr la reelección, bien porque piense que ese es su deber). De este modo, con el paso del tiempo los diputados tienden a alinear sus opiniones con las de su distrito (Miller y Stokes, 1963: 51).

En este caso, cuando los representantes están influenciados por sus electores, puede suceder que los diputados de circunscripciones menos desarrolladas no prefieran la honradez sobre el carisma, porque aquella característica no está en las preferencias de su electorado. Si esto fuera así (hipótesis 2) los diputados elegidos en circunscripciones

11 Para Miller y Stokes son necesarias tres condiciones para que los ciudadanos puedan ejercer influencia sobre los representantes. "Primero el voto del diputado en la Cámara debe coincidir con sus propias ideas o con lo que cree que son las ideas de su distrito. En segundo lugar, las creencias que dirigen los actos del diputado deben corresponderse aunque sea de forma imperfecta con las opiniones del distrito. [...] Finalmente, los ciudadanos deben tener en cuenta las visiones del candidato cuando eligen un representante" (1963: 51). 
con niveles educativos de su población inferiores y menos desarrolladas no diferenciarán entre el carisma y la honradez, o la capacidad. Asimismo, en departamentos con altos niveles de educación los diputados preferirán con mayor probabilidad la honradez y la capacidad sobre el carisma, ya que se corresponden con las preferencias de su electorado.

Finalmente, en el análisis se considera que la opción por unas u otras características pueda depender tanto del nivel de sofisticación política del diputado como del grado de sofisticación del electorado de su circunscripción. En este caso (hipótesis 3 ) se espera que sean los políticos más educados los que prefieran la honradez y la capacidad con más probabilidad en aquellas circunscripciones con un desarrollo alto que en los distritos con un nivel de desarrollo menor.

\section{LOS DATOS Y EL ANÁLISIS: LAS CUALIDADES DE LOS POLÍTICOS EN AMÉRICA LATINA}

Para comprobar estas hipótesis se utilizan los datos del Proyecto Elites Parlamentarias Latinoamericanas (PELA) de la Universidad de Salamanca. En el análisis se incluyen las entrevistas realizadas mediante cuestionario y de forma personal a 1.379 diputados, de 10 países y 16 períodos legislativos (ver Anexo I). El cuestionario aplicado en las dos últimas rondas de entrevistas incluye preguntas ${ }^{12}$ referidas a las características de un buen político, pero las características más importantes para ganar elecciones solo están en la última ronda. Sin embargo, ya que las categorías incluidas en ambas preguntas son distintas, los análisis se han realizado con varias categorizaciones diferentes de la variable dependiente. ${ }^{13}$

En la Tabla 1 se muestran las principales características que según los legisladores son deseables en un buen político. En los casos de estudio aquí incluidos, al igual que sucede para los ciudadanos, la honradez es la cualidad más importante que debe tener un buen político. Los datos medios muestran que 49,3\% de los diputados elige la honradez como la característica más importante y en segundo lugar aparece el carisma con 16,3\% de las respuestas. Sin embargo, aunque la honradez sea la característica más valorada sin excepción, los porcentajes varían entre unos casos y otros de forma importante; encontrando el apoyo máximo en El Salvador (65,3\%) y el mínimo en Colombia (31,3\%). Como cabía esperar, a tenor de las referencias de otros autores sobre la opinión de los políticos, la segunda característica más importante en un buen político no es la capacidad como sucede entre los ciudadanos. La cualidad que ocupa el segundo espacio es el carisma y sucede en 10 de los 16 casos. Además, en los cinco casos restantes solo en tres (México en las dos olas y Paraguay) la capacidad está mejor valorada que el carisma. Por tanto, en las 16 legislaturas analizadas la honradez es el aspecto más importante que ha de tener un buen político, y en 10 de 16 el carisma es el segundo elemento más importante. Estos datos ponen de manifiesto la relevancia que el carisma tiene entre

12 Las preguntas usadas en el análisis son de tipo cerrado, si bien en el cuestionario aparecen algunas preguntas abiertas pero que no están relacionadas con esta temática.

13 En el Anexo II se detallan todas las variables incluidas en los análisis así como las categorías de respuesta. 
los políticos y las divergencias entre estos y la ciudadanía, donde la capacidad es la segunda característica mejor valorada.

Cuando la pregunta que se realiza a los diputados tiene que ver con las características que sirven para ganar elecciones las categorías más señaladas son el carisma $(25,2 \%)$ y el liderazgo (28,9\%), que de forma conjunta sobrepasan el $50 \%$ de las respuestas (ver Tabla 2). ${ }^{14}$ La honradez, que en la anterior pregunta es la opción más señalada, pasa a ser la tercera opción con $20 \%$ de las respuestas. Si se observan los datos para cada uno de los países solo en dos de ellos, Honduras y Perú, la honradez tiene un porcentaje de respuesta mayor que el carisma.

Tabla 1. Características más importantes que debe tener un buen político (porcentajes)

\begin{tabular}{lrccccc}
\hline \multicolumn{1}{c}{ País } & Carisma & Honradez & Capacidad & Otros & Total & (n) \\
\hline Costa Rica & 19,3 & 59,6 & 7,0 & 14,0 & 100 & $(57)$ \\
Honduras & 24,2 & 52,7 & 4,4 & 18,7 & 100 & $(91)$ \\
El Salvador & 13,9 & 65,3 & 6,9 & 13,9 & 100 & $(72)$ \\
Colombia & 12,8 & 38,6 & 6,0 & 42,6 & 100 & $(107)$ \\
Perú & 14,0 & 44,1 & 14,0 & 28,0 & 100 & $(93)$ \\
Bolivia & 26,0 & 42,5 & 6,3 & 25,2 & 100 & $(96)$ \\
México & 3,9 & 54,8 & 14,1 & 27,3 & 100 & $(128)$ \\
Guatemala & 32,2 & 40,1 & 3,0 & 24,7 & 100 & $(97)$ \\
Paraguay & 2,8 & 50,0 & 6,9 & 40,3 & 100 & $(72)$ \\
El Salvador b & 17,6 & 64,7 & 5,9 & 11,8 & 100 & $(68)$ \\
Panamá & 35,9 & 39,1 & 7,8 & 17,2 & 100 & $(64)$ \\
Honduras b & 27,5 & 50,5 & 5,5 & 16,5 & 100 & $(91)$ \\
Costa Rica b & 14,5 & 60,0 & 9,1 & 16,4 & 100 & $(55)$ \\
México b & 1,8 & 53,0 & 15,5 & 29,7 & 100 & $(97)$ \\
Colombia b & 10,2 & 31,3 & 3,4 & 55,1 & 100 & $(89)$ \\
Perú b & 12,1 & 55,5 & 6,1 & 26,3 & 100 & $(83)$ \\
\hline Media & 16,3 & 49,3 & 7,8 & 26,6 & 100 & $(1.360)$ \\
\hline
\end{tabular}

Fuente: PELA (1994-2016). Nota: Bajo la categoría "otros" se incluyen las siguientes respuestas: experiencia en cargos anteriores; gestor eficiente y eficaz, resolutivo; deliberante, dialogante; comprometido con su labor política; amplia formación educativa y conocimientos técnicos; que cumpla sus promesas electorales.

b: ver legislaturas en Anexo I.

14 Otras visiones complementarias sobre la carrera y el éxito político pueden consultarse en Alcántara (2012), Laguna (2010) y Roa Suárez (2005). 
Tabla 2. Características más importantes de un político para ganar elecciones (porcentajes)

\begin{tabular}{lrrrrrrr}
\hline & Formación & $\begin{array}{c}\text { Experiencia } \\
\text { política }\end{array}$ & Honradez & Carisma & $\begin{array}{c}\text { Liderazgo } \\
\text { político }\end{array}$ & Total & (n) \\
\hline Colombia b & 18,6 & 30,2 & 14,0 & 9,3 & 27,9 & 100 & $(86)$ \\
Costa Rica b & 1,8 & 5,5 & 21,8 & 30,9 & 40,0 & 100 & $(55)$ \\
El Salvador b & 2,9 & 10,3 & 17,6 & 17,6 & 51,5 & 100 & $(68)$ \\
Honduras b & 17,6 & 23,1 & 29,7 & 24,2 & 5,5 & 100 & $(91)$ \\
México b & 3,2 & 8,4 & 21,1 & 40,0 & 27,4 & 100 & $(95)$ \\
Panamá & 3,1 & 10,9 & 15,6 & 42,2 & 28,1 & 100 & $(64)$ \\
Perú b & 17,9 & 14,3 & 20,2 & 15,5 & 32,1 & 100 & $(84)$ \\
\hline Media & 10,1 & 15,5 & 20,3 & 25,2 & 28,9 & 100 & $(543)$ \\
\hline
\end{tabular}

Fuente: PELA (1994-2016).

b: ver legislaturas en Anexo I.

A pesar de las cautelas que hay que tomar al ser dos preguntas con distintas categorías queda claro que la importancia de la honradez y del carisma como características de valoración se invierte cuando se pasa de considerar lo que es propio de un buen político, a considerar lo que es necesario para ganar elecciones. En general, la honradez pasa a ser una característica de tercer orden.

Con estos datos en la mano se han realizado dos análisis diferenciados, el primero tomando como variable dependiente las características de un buen político y el segundo a partir de las respuestas a la pregunta sobre qué características son propias de un político ganador. El objetivo es identificar en qué medida el grado de sofisticación política de los diputados y de su electorado altera las percepciones y la ordenación que se ha descrito anteriormente. Los análisis comparten las mismas variables independientes, 12 en todos los modelos (ver Anexo II). Para poder medir las diferencias en el grado de sofisticación de los políticos se tiene en cuenta el nivel de formación educativa y la experiencia política. El nivel educativo se recoge en una sola pregunta, sin embargo, la experiencia política se basa en cuatro aspectos: la experiencia legislativa previa, la experiencia en cargos de partido, en puestos de designación y, finalmente, si el diputado tiene experiencia anterior en el ejercicio de otros cargos de elección popular. El nivel educativo muestra el grado de adquisición de conocimientos formales. Por su parte, la experiencia política previa es importante, ya que políticos de calidad pueden tratar de construir una reputación mediante elecciones de menor rango (Galasso y Nannicini, 2011). Además, los diputados con más conocimientos y más formados pueden converger con las ideas de sus electores, como sostienen Miller y Stokes (1963), llegando a mayores niveles de congruencia.

Para poder medir las diferencias en el grado de sofisticación de los ciudadanos se ha utilizado el índice de desarrollo humano (IDH) elaborado por el PNUD. Este índice está 
basado en los niveles de desarrollo de cada país en tres ámbitos: la educación, la salud y la riqueza. Lamentablemente la organización no ofrece este dato para todos los países latinoamericanos a nivel subnacional, de forma que fuera posible relacionar al electorado y al diputado en su circunscripción en todos los casos. Debido a la limitada disposición de esta información el número de países incluido en el análisis no comprende los 18 casos que se incluyen en el proyecto PELA. Menor aún es el número de casos en los que la información subnacional del índice se ofrece de forma desagregada distinguiendo el perfil educativo, sanitario y de riqueza. Por esta razón se ha mantenido la información del índice original que da una visión global del grado de vulnerabilidad que tienen los ciudadanos en un determinado territorio para los 10 países donde la información de la distribución territorial del índice está disponible y es comparable con la división geográfica electoral. ${ }^{15}$

Junto a las variables independientes propias del diputado y las relativas a los electores del departamento del diputado, también se han incluido las siguientes variables de control: edad, género, si el diputado es parte del partido de gobierno o se encuentra en la oposición, posición ideológica en la escala izquierda-derecha, ${ }^{16}$ nivel de ingresos y, finalmente, si otros miembros de su familia se dedican o se han dedicado a la política.

\section{El buen político y la sofisticación política de los diputados}

Como anteriormente se señalaba, si es cierto que el nivel de sofisticación política de los individuos afecta la elección de las características más valoradas en un político, y si este impacto se produce como la literatura ha propuesto hasta ahora, se espera que los diputados más sofisticados (ya tengan mayor educación o más experiencia política) prefieran la honradez por encima del carisma. También se espera que, si los diputados están influidos por su electorado, los representantes de circunscripciones más desarrolladas también elijan con más probabilidades la honradez que el carisma. Asimismo, si la sofisticación del diputado y las características del departamento influyen de forma conjunta, la preferencia por la honradez será mayor cuando un diputado con alto nivel educativo represente a un departamento más desarrollado que cuando proceda de una circunscripción con un IDH menor.

También hubiera sido pertinente incluir información procedente de encuestas de opinión pública como la realizada, por ejemplo, por el Barómetro de las Américas de la Universidad de Vanderbilt, pero la representatividad territorial de sus encuestas no coincide con la división electoral. Una aplicación de estos datos puede encontrarse en Mondak et al. (2011).

16 Si bien aquí se considera la variable en su versión izquierda-derecha otros autores han tomado la distancia respecto de la media como medida ideológica más relevante. Según Stone y Simas (2010), los diputados con una ventaja basada en características personales están ideológicamente más cercanos a las preferencias de sus distritos, mientras que los desafiantes en desventaja tienden a tener posiciones ideológicas más extremas. En una situación en donde no existe diferencia ideológica aquel candidato con mayor ventaja en los aspectos personales fuerza al candidato en desventaja a crear una diferencia ideológica para que la decisión del elector no se base únicamente en las características personales. De este modo el candidato en desventaja trata de ganar votos asumiendo posiciones ideológicas más extremas y haciendo que el candidato con ventaja personal sea a su vez el que mantiene una mayor congruencia con el electorado de su distrito. 
Porque la variable dependiente contiene más de dos categorías, no es posible emplear la regresión logística para el análisis estadístico, pero sí una extensión de esta técnica denominada regresión logística multinomial. De esta forma es posible testar la relación entre una variable dependiente con más de dos categorías y las variables independientes que se determinen, ya sean estas nominales o continuas. Esta técnica compara cada una de las categorías de la variable dependiente con una categoría fija que el usuario establece como categoría de referencia, en este caso el carisma. Así, en la Tabla 3 se han realizado cuatro modelos distintos, con distintas categorizaciones de la variable dependiente. En el primero la variable dependiente tiene tres categorías, en el segundo cuatro, en el tercero cinco y en el cuarto modelo hay cuatro categorías. Los resultados del análisis permiten establecer qué variables independientes hacen distintas a dos categorías de la variable dependiente: en el modelo 1, por ejemplo, la variable "novato" tiene un impacto distinto en aquellos diputados que creen que un buen diputado debe ser honrado que en los que piensan que un buen diputado debe tener carisma. Además de identificar qué variables son significativas los resultados permiten calcular en qué medida las dos categorías son distintas mediante el EXP(B). Cuando el valor del EXP(B) es mayor que uno la variable está relacionada de forma positiva con esa categoría en comparación con la variable de referencia. En caso de que sea menor que uno, esa variable está relacionada negativamente con esa categoría, si se compara con la categoría de referencia.

En la Tabla 3 se recogen los resultados de los distintos análisis multinomiales realizados para la variable de las cualidades de un buen político. Los modelos dan como resultado la significación de seis variables independientes: la experiencia legislativa y de partido, el índice de desarrollo humano de la circunscripción, la ideología, la pertenencia al partido de gobierno y los ingresos. Al contrario de lo esperado, el nivel educativo de los entrevistados no tiene un impacto estadísticamente significativo. Una posible explicación puede ser la poca variación de los niveles educativos de los representantes políticos, que es mucho menor que entre la ciudadanía, siendo muy pequeño el número de diputados sin estudios o con estudios primarios (Martínez Rosón, 2009).

Otras variables relacionadas con la expertise política sí resultan significativas. En la elección de las características más valoradas la experiencia legislativa previa es una variable que sirve para distinguir las preferencias de los diputados sobre las cualidades que definen a un buen político. La probabilidad de que los diputados prefieran la honradez al carisma es mayor entre los diputados novatos que entre los diputados con legislaturas previas. En los distintos modelos estadísticos se ha encontrado que la honradez es preferida al carisma $70 \%$ más entre los diputados novatos que entre los experimentados. Igual sucede en el caso de la capacidad, los diputados noveles prefieren esta característica por encima del carisma en mayor medida que los diputados senior: los diputados sin experiencia legislativa previa prefieren la capacidad al carisma en el doble de ocasiones que los diputados con experiencia.

Más allá de la elección entre honradez y carisma también se producen diferencias debidas a la experiencia política previa fuera del legislativo. Así, la experiencia partidista de los diputados que han asumido cargos de organización interna modifica la imagen 


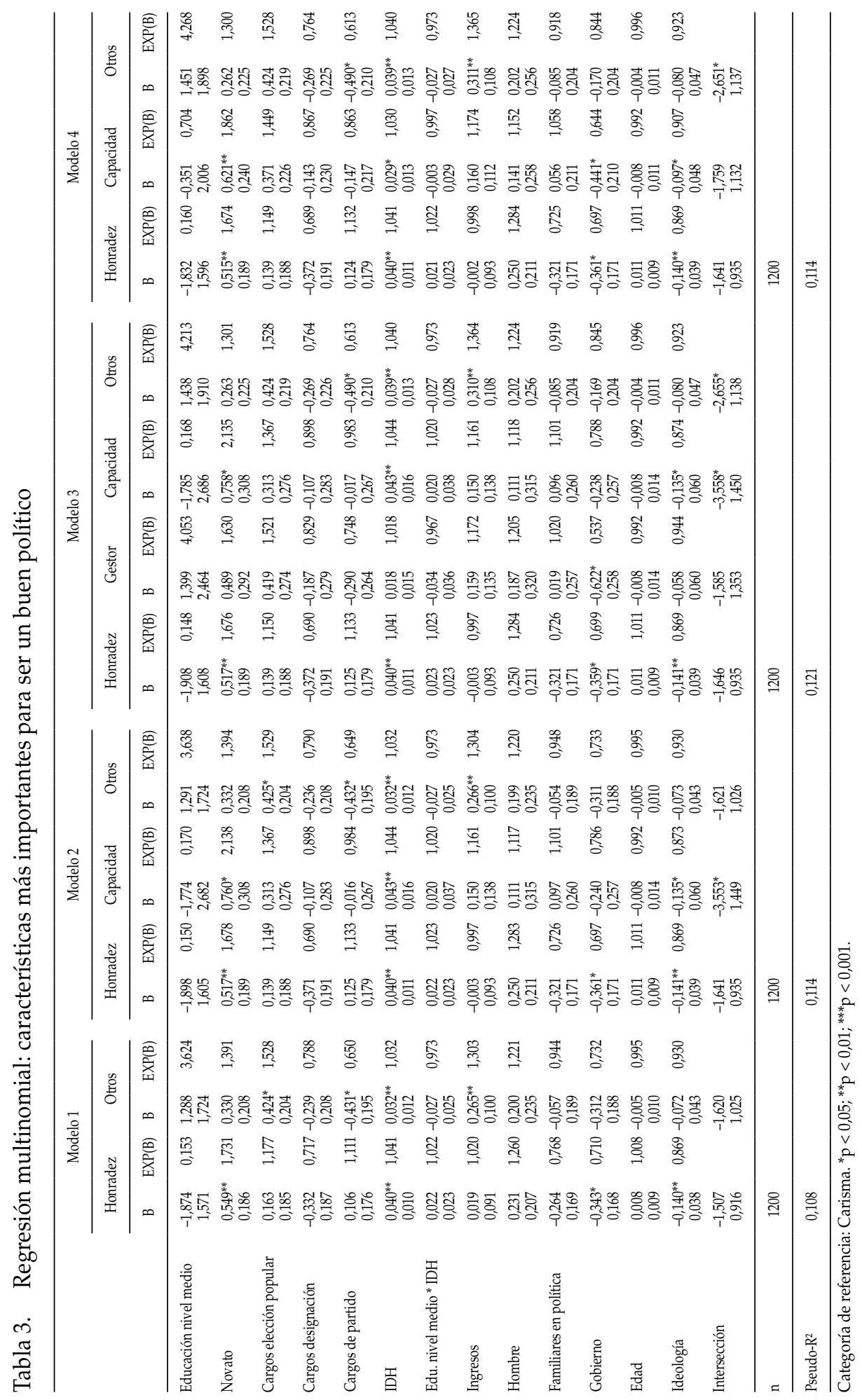




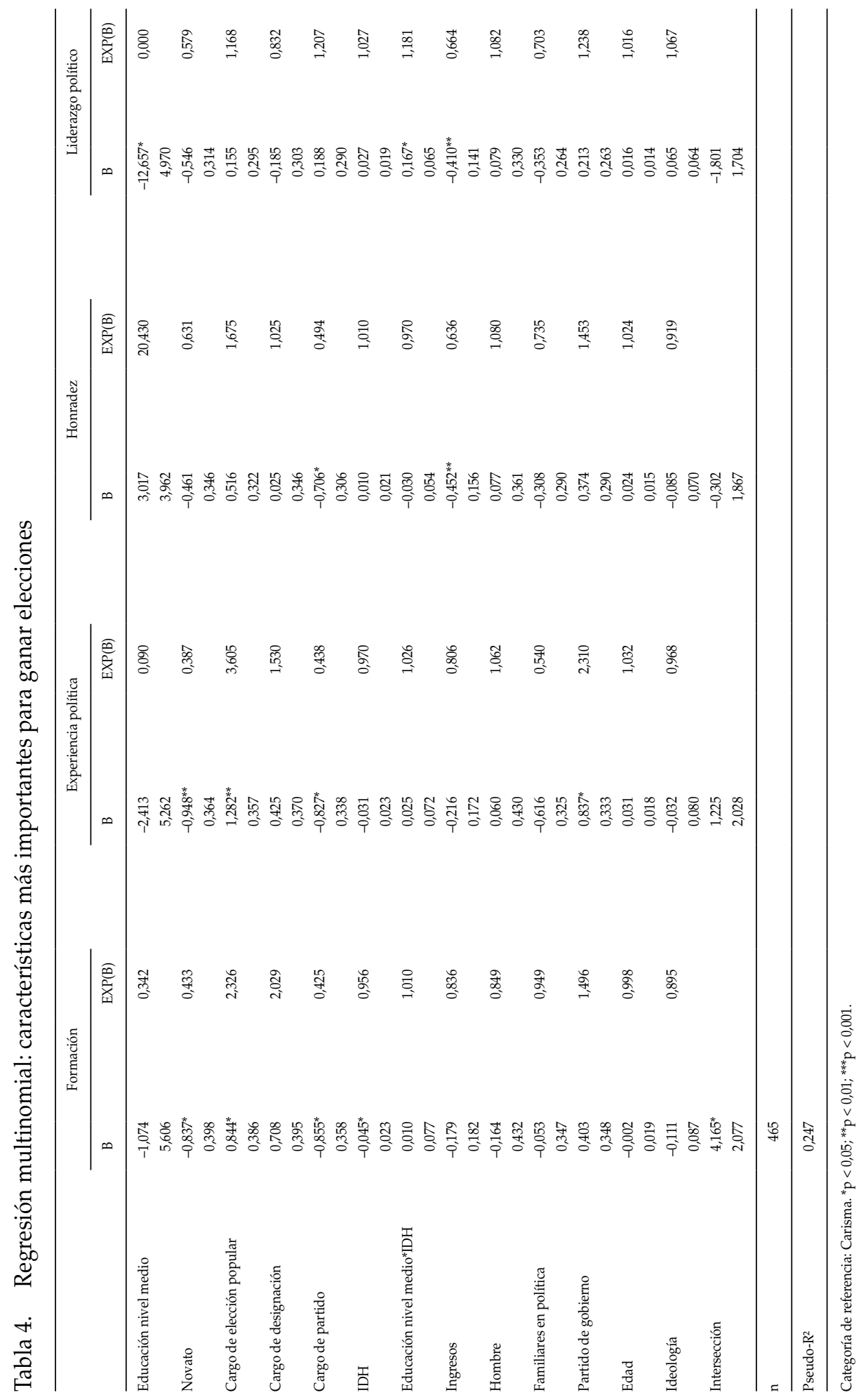


que tienen de un buen político. Los diputados que se han desempeñado en cargos de partido eligen características minoritarias con menor probabilidad que los diputados sin experiencia. Es decir, los diputados que han asumido cargos internos valoran el carisma hasta $60 \%$ más que los diputados sin cargos en comparación con la categoría de otras características.

El impacto de ambas variables, la experiencia legislativa previa y la experiencia en cargos de partido se produce en un sentido diferente al que cabría esperar. Si como aquí se ha definido, un diputado con mayor experiencia es un diputado con un mayor nivel de sofisticación política, esta no está relacionada con una mayor preferencia por la honradez o la capacidad. Mientras que entre la ciudadanía son los más sofisticados políticamente quienes tienen unas preferencias más claras por la honradez y la capacidad, entre los legisladores el mayor nivel de sofisticación política se relaciona con la preferencia por el carisma. En definitiva, la experiencia política, en el partido o en la Cámara, desmitifica el valor de la capacidad y de la honradez a favor del carisma como características deseables en un buen político, mientras que la falta de experiencia legislativa o partidista está relacionada con un mayor apoyo a la honradez y la capacidad como cualidades deseables en un buen político.

Por otro lado, con lo que respecta al índice de desarrollo humano, como variable que representa el grado de desarrollo del departamento o de la circunscripción del diputado, existe una relación con todas las categorías de la variable dependiente. Cuando aumenta el desarrollo humano del departamento aumenta la preferencia por la honradez, la capacidad y todas las demás características en detrimento del carisma del político. Por cada mejora en el desarrollo humano de una unidad (medida aquí de 0 a 100), disminuye la preferencia por el carisma en $4 \%$ frente a la honradez y también frente a la capacidad como características propias de un buen político.

Esto significa que los diputados no son ajenos a las características de su audiencia. Respondiendo de acuerdo con lo que estudios previos han resaltado para los ciudadanos, entre los legisladores de circunscripciones menos desarrolladas, donde se espera un menor nivel de sofisticación política de los ciudadanos, las preferencias por el carisma son más probables. De forma complementaria, en los departamentos más desarrollados los diputados eligen con más probabilidad la honradez que el carisma. Ya sea de manera consciente o de manera inconsciente los diputados se comportan como lo hace su electorado.

\section{El político ganador y la sofisticación política de los diputados}

El análisis multinomial realizado con la variable referida a las características más relevantes para ganar elecciones se realiza con el mismo número de variables independientes que el análisis previo. Además, en la variable dependiente se mantienen las cinco categorías originales de la pregunta. Los resultados muestran que la experiencia en cargos de partido y en cargos de elección popular, el IDH, el nivel educativo, la interacción entre la educación y el nivel de desarrollo de la circunscripción electoral y los ingresos tienen impacto en las características que los diputados seleccionan como elementales para ganar elecciones. 
En general hay que señalar que la probabilidad de la elección del carisma frente a otras cualidades es similar entre los diputados que cuentan con experiencia en cargos de partido o es su primera vez en el Parlamento. Para estos diputados la probabilidad de elegir el carisma como la característica más importante para ganar elecciones es mayor que para los diputados con más experiencia legislativa y sin experiencia partidista. Aquellos eligen la honradez con una probabilidad del 50\% menos que el carisma, la experiencia política entre $56 \%$ y $60 \%$ menos y la formación $56 \%$ menos.

Aquellos diputados que han desempeñado previamente cargos de elección popular y los que pertenecen al partido de gobierno tienen una visión diferente de la importancia del carisma. Al contrario de lo que sucede con otros diputados, en estos casos las cualidades formativas y de experiencia política previa son más importantes que el carisma para ganar elecciones. Aquellos diputados que han tenido un cargo de elección popular antes de llegar al Parlamento eligen la formación el doble de veces que el carisma y eligen la experiencia política previa el triple de veces. Por su parte, la probabilidad de que los diputados del partido de gobierno prefieran la experiencia política dobla la probabilidad de que elijan el carisma.

Esto significa que todas las variables que se han considerado aquí para valorar el grado de sofisticación política de los diputados no afectan la selección de características en la misma dirección. Los datos muestran que cuanto mayor sea la experiencia electoral y de gobierno del diputado menos importancia se le da al carisma, mientras que por el contrario, el carisma es una cualidad fundamental para ganar elecciones para aquellos diputados cuya experiencia legislativa es menor o su experiencia no ha estado relacionada con procesos electorales.

\section{CONCLUSIONES}

La literatura centrada en el estudio de las características personales de los políticos y de los candidatos ha sido siempre cautelosa en descartar el carisma como factor relevante en la valoración de los políticos. Mientras que los distintos estudios que se centraban en datos recabados entre los ciudadanos y en otros ámbitos regionales han llegado a la conclusión de que la honradez y la competencia son las dos cualidades con más peso en las valoraciones realizadas por los ciudadanos, los políticos subrayaban la importancia del carisma. En este trabajo se muestra que estas dos características no son las más relevantes desde la propia perspectiva de los legisladores latinoamericanos. Aunque la honradez es la cualidad más importante, el segundo lugar lo ocupa el carisma. Asimismo, cuando se pregunta por cuáles son las características más importantes para ganar elecciones las dos respuestas principales son el carisma y el liderazgo, pasando la honradez a un tercer lugar. Esto pone de manifiesto la diferencia que existe entre ser un buen político y ser un político que gana elecciones. Cada una de estas dos cuestiones requiere de cualidades diferentes a los ojos de los diputados. Este resultado tiene implicaciones en todos aquellos estudios que tratan de relacionar las cualidades personales con el voto. Ser un buen candidato no parece corresponderse al cien por cien con ser el candidato 
más votado. Según los diputados latinoamericanos un político honrado es un buen político, pero un diputado que es carismático es el que finalmente gana la contienda electoral. La diferencia entre lo que es bueno y lo que es electoralmente efectivo explica la discrepancia que anteriormente han advertido otros investigadores. Los datos muestran muy claramente este antagonismo para los diputados novatos. Estos creen que la honradez es una buena cualidad, con $70 \%$ más de probabilidades que un diputado senior. Sin embargo, los novatos también creen que el carisma es una buena calidad para ganar elecciones con $60 \%$ más de probabilidad que los diputados reelegidos.

Por otro lado, distintos análisis han identificado qué variables como el grado de conocimientos políticos alteran estas preferencias, siendo que aquellos ciudadanos políticamente menos sofisticados responden mejor a características como el carisma mientras que los ciudadanos políticamente más sofisticados responden mejor a cualidades como la honradez. Aunque esto se ha comprobado en el ámbito de los electores poco se sabe de cómo perciben y actúan los políticos ante este hecho. El objetivo de este trabajo ha sido comprobar si el nivel de formación y los conocimientos políticos adquiridos a lo largo de su carrera política también alteran la percepción que tienen los diputados sobre qué características son más valoradas en un político y cuáles sirven mejor para ganar elecciones, si es el grado de conocimientos políticos de su electorado el que modifica sus preferencias, o si ambas son ciertas.

Al contrario de lo que sucede entre los ciudadanos el nivel educativo no establece diferencias importantes entre las cualidades que valoran los diputados en un buen político. Sin embargo, otras variables relacionadas con la experiencia política sí muestran resultados interesantes. La importancia de la honradez frente al carisma es mayor entre los diputados novatos. Además, los diputados responden en concordancia con su electorado siendo mayor el apoyo a la honradez que al carisma en aquellos departamentos donde el desarrollo de su población es mayor. Por tanto, una mayor sofisticación política del diputado va vinculada a una preferencia mayor por el carisma, contradiciendo así lo que sucede en la ciudadanía. Pero sorprendentemente, una mayor sofisticación del electorado está relacionada con un mayor apoyo a la honradez y a la capacidad. De esta forma los resultados están en consonancia con los encontrados con Miller y Stokes (1963), que defienden la influencia de los ciudadanos sobre las élites.

El impacto de los conocimientos políticos también afecta a la selección de las variables más importantes para ganar elecciones. En este caso parece que los diputados que han ejercido labores en otros cargos de elección popular o pertenecen al partido de gobierno dan menos importancia al carisma, mientras que los diputados de oposición y los novatos valoran más el carisma. Estos datos ponen de manifiesto que no todas las experiencias políticas que mejoran los conocimientos de los políticos actúan en la misma dirección. Algunas experiencias hacen que el carisma sea más valorado y otras hacen disminuir su importancia a los ojos de los diputados. La práctica de gobierno y legislativa así como la práctica electoral tienen distintas implicaciones, siendo que cuanto mayor sea su ejercicio menos relevancia se le otorga al carisma como cualidad importante para ganar elecciones. Estos resultados están en consonancia con los hallazgos previos de otros investigadores 
que ponen de manifiesto el deterioro de los beneficios del carisma que en el largo plazo se produce ante un mal desempeño político (Merolla y Zechmeister, 2011).

En definitiva, se puede concluir que cuanto mayor es la experiencia electoral de un diputado, cuando su carrera política ha dependido en mayor medida del favor de las urnas, menor es la importancia que le otorgan al carisma como factor fundamental para ganar elecciones. En este sentido cabe precisar, por tanto, que entre los legisladores la sofisticación política diferencia las preferencias de los diputados pero solo una trayectoria política electoral está vinculada al rechazo del carisma.

Estos resultados están en consonancia con las conclusiones de Miller y Stokes (1963). Si bien la congruencia entre la opinión de los representantes y de los representados sobre qué características definen a un buen diputado no es coincidente (sobre todo entre diputados con mayor expertise), existe una mayor congruencia entre la opinión de los ciudadanos y lo que los representantes piensan que demandan, es decir, las características que sirven para ganar elecciones; y la influencia de los ciudadanos sobre las élites es mayor entre los diputados con mayor experiencia.

\section{REFERENCIAS}

Abney, Ronni, James Adams, Michael Clark, Malcolm Easton, Lawrence Ezrow, Spyros Kosmidis y Anja Neundorf. 2013. "When Does Valence Matter? Heightened Valence Effects for Governing Parties During Election Campaigns". Party Politics 19 (1): 61-82.

Abramowitz, Alan I. 1991. "Incumbency, Campaign Spending, and the Decline of Competition in US House Elections". Journal of Politics 53 (1): 34-56.

Alcántara, Manuel. Director. 1994-2016. "Proyecto de Elites Parlamentarias Latinoamericanas (PELA)". Salamanca: Universidad de Salamanca.

Alcántara, Manuel. 2012. El oficio del político. Madrid: Tecnos.

Canache, Damarys, Jeffery J. Mondak y Ernesto Cabrera. 2000. “Voters and the Personal Vote: A Counterfactual Simulation". Political Research Quarterly 53 (3): 663-676.

Clark, Michael. 2009. "Valence and Electoral Outcomes in Western Europe, 1976-1998". Electoral Studies 28 (1): 111-122.

Finan, Frederico y Claudio Ferraz. 2005. “Reelection Incentives and Political Corruption: Evidence from Brazilian Audit Reports". Ponencia presentada en el Annual Meeting of the American Agricultural Economics Association, julio 24-27, Providence, RI.

Fiorina, Morris P. 1981. Retrospective Voting in American Elections. New Haven: Yale University Press.

Fisher, Stephen D., Laurence Lessard-Phillips, Sara Hobolt y John Curtice. 2008. “Disengaging Voters: Do Plurality Systems Discourage the Less Knowledgeable from Voting?". Electoral Studies 27 (1): 89-104.

Funk, Carolyn L. 1996. "The Impact of Scandal on Candidate Evaluations: An Experimental Test of the Role of Candidate Traits". Political Behavior 18 (1): 1-24.

Funk, Carolyn L. 1997. "Implications of Political Expertise in Candidate Trait Evaluations". Political Research Quarterly 50 (3): 675-697.

Funk, Carolyn L. 1999. "Bringing the Candidate into Models of Candidate Evaluation". Journal of Politics 61 (3): 700-720.

Galasso, Vinzenco y Tommaso Nannicini. 2011. "Competing on Good Politicians". American Political Science Review 105 (1): 79-99.

Gordon, Stacy B. y Gary M. Segura. 1997. "Cross-National Variation in the Political Sophistication of Individuals: Capability or Choice?". The Journal of Politics 59 (1): 126-147. 
Green, Jane. 2007. "When Voters and Parties Agree: Valence Issues and Party Competition". Political Studies 55 (3): 629-655.

Groseclose, Tim. 2001. "A Model of Candidate Location When One Voter Has a Valence Advantage". American Journal of Political Science 45 (4): 862- 886.

Hall, Melinda G. y Chris W. Bonneau. 2006. "Does Quality Matter? Challengers in [US] State Supreme Court Elections". American Journal of Political Science 50 (1): 20-33.

Hayes, Danny. 2005. "Candidate Qualities through a Partisan Lens: A Theory of Trait Ownership". American Journal of Political Science 49 (4): 908-923.

Huckfeldt, Robert, Jeffery J. Mondak, Michael Craw y Jeanette Morehouse Mendez. 2005. “Making Sense of Candidates: Partisanship, Ideology, and Issues as Guides to Judgment". Cognitive Brain Research 23 (1): 11-23.

Jerit, Jennifer, Jason Barabas y Toby Bolsen. 2006. "Citizens, Knowledge, and the Information Environment". American Journal of Political Science 50 (2): 266-282.

Kinder, Donald R., Mark D. Peters, Robert P. Abelson y Susan T. Fiske. 1980. "Presidential Prototypes". Political Behavior 2 (4): 315-337.

Kinder, Donald R. 1983. Presidential Traits. Ann Arbor: University of Michigan.

Krassa, Michael A. 1990. "Political Information, Social Environments, and Deviants". Political Behavior 12 (4): 315-330.

Kuklinski, James H. y Paul J. Quirk. 2001. "Conceptual Foundations of Citizen Competence". Political Behavior 23 (3): 285-311.

Kuklinski, James H., Paul J. Quirk, Jennifer Jerit y Robert F. Rich. 2001. “Political Environment and Citizen Competence". American Journal of Political Science 45 (2): 410-424.

Kulisheck, Michael R. y Jeffery J. Mondak. 1996. “Candidate Quality and the Congressional Vote: A Causal Connection?". Electoral Studies 15 (2): 237-253.

Laguna, Antonio. 2010. Las claves del éxito político. ¿Por qué votan los ciudadanos? Barcelona: Ediciones Península.

Lau, Richard R. y David P. Redlawsk. 2001. "Advantages and Disadvantages of Cognitive Heuristics in Political Decision Making". American Journal of Political Science 45 (4): 951-971.

Lau, Richard R. y David P. Redlawsk. 2008. "Older but Wiser? Effects of Age on Political Cognition”. The Journal of Politics 70 (1): 168-185.

Mainwaring, Scott y Mariano Torcal. 2005. “La institucionalización de los sistemas partidistas y la teoría del sistema partidista después de la tercera ola democratizadora". América Latina Hoy 41: 141-173.

Martínez Rosón, María del Mar. 2009. “La ambición política en situaciones adversas: contextos institucionales y personales". Revista de Estudios Políticos 146: 113-148.

Martínez Rosón, María del Mar. 2011. “Ambición política y lealtad. Influencia sobre el comportamiento político". Política y Gobierno 18 (2): 231-264.

McCurley, Carl y Jeffery J. Mondak. 1995. “Inspected by \#1184063113: The Influence of Incumbents Competence and Integrity in U.S. House Elections". American Journal of Political Science 39 (4): 864-885.

Merolla, Jennifer L. y Elizabeth J. Zechmeister. 2011. "The Nature, Determinants, and Consequences of Chávez's Charisma: Evidence from a Study of Venezuelan Public Opinion". Comparative Political Studies 44 (1): 28-54.

Miller, Warren E. y Donald E. Stokes. 1963. "Constituency Influence in Congress". The American Political Science Review 57 (1): 45-56.

Moncrief, Gary F., Peverill Squire y Malcolm E. Jewell. 2001. Who Runs for the Legislature? Candidates and Recruitment in the States. Upper Saddle River: Prentice-Hall.

Mondak, Jeffery J. 1995. "Competence, Integrity, and the Electoral Success of Congressional Incumbents". The Journal of Politics 57 (4): 1043-1069.

Mondak, Jeffery J., Damarys Canache, Mitchell A. Seligson y Matthew V. Hibbing. 2011. “The Participatory Personality: Evidence from Latin America". British Journal of Political Science 41 (1): 211-221.

Page, Benjamin I. 1978. Choices and Echoes in Presidential Elections. Chicago: University of Chicago Press. Popkin, Samuel L. 1991. The Reasoning Voter: Communication and Persuasion in Presidential Campaigns. Chicago: University of Chicago Press. 
Prysby, Charles. 2008. "Perceptions of Candidate Character Traits and the Presidential Vote in 2004". Political Science and Politics 41 (1): 115-122.

Roa Suárez, Hernando. 2005. El liderazgo político. Análisis de casos. Bogotá: Fondo Editorial, UPN.

Shugart, Matthew S., Melody Ellis Valdini y Kati Suominen. 2005. “Looking for Locals: Voter Information Demands and Personal Vote-earning Attributes of Legislators under Proportional Representation". American Journal of Political Science 49 (2): 437-449.

Squire, Peverill. 1993. "Professionalization and Public Opinion of State Legislatures". Journal of Politics 55 (2): 479-491.

Squire, Peverill. 1998. "Membership Turnover and the Efficient Processing of Legislation". Legislative Studies Quarterly 23 (1): 23-32.

Squire, Peverill y Keith E. Hamm. 2005. 101 Chambers: Congress, State Legislatures, and the Future of Legislative Studies. Columbus: Ohio State University Press.

Stokes, Donald E. 1963. "Spatial Models of Party Competition". The American Political Science Review 57 (2): 368-377.

Stone, Walter J., Sandy Maisel y Cherie D. Maestas. 2004. “Quality Counts: Extending the Strategic Politician Model of Incumbent Deterrence". American Journal of Political Science 48 (3): 479-495.

Stone, Walter J. y Elizabeth N. Simas. 2010. "Candidate Valence and Ideological Positions in U.S. House Elections". American Journal of Political Science 54 (2): 371-388.

Sullivan, John L., John H. Aldrich, Eugene Borgida y Wendy Rahn. 1990. “Candidate Appraisal and Human Nature: Man and Superman in the 1984 Election". Political Psychology 11 (3): 459-484.

Welch, Susan y John Hibbing. 1997. "The Effect of Charges of Corruption on Voting Behavior in Congressional Elections, 1982-90". Journal of Politics 59 (1): 226-239.

Zaller, John R. 1992. The Nature and Origins of Mass Opinion. New York: Cambridge University Press. 


\section{ANEXOS}

\section{Relación de países y legislaturas incluidas en los análisis}

Los diputados entrevistados se clasificaron en un número variable de estratos (en función del número de partidos con representación parlamentaria presentes), estableciéndose una afijación proporcional para la distribución de la muestra. La selección se realizó de forma aleatoria entre los diputados pertenecientes a cada uno de los estratos.

\begin{tabular}{lccccccc}
\hline \multicolumn{1}{c}{ País } & $\begin{array}{c}\text { Número } \\
\text { de } \\
\text { Estudio }\end{array}$ & $\begin{array}{c}\text { Tamaño } \\
\text { de la } \\
\text { Cámara }\end{array}$ & $\begin{array}{c}\text { Entrevistas } \\
\text { realizadas }\end{array}$ & $\%$ & Período & $\begin{array}{c}\text { V.D. } \\
\text { Buen } \\
\text { político }\end{array}$ & $\begin{array}{c}\text { V.D. } \\
\text { Ganador }\end{array}$ \\
\hline Bolivia & 62 & 130 & 98 & 75,4 & $2006-2010$ & x & \\
Colombia & 59 & 166 & 107 & 64,5 & $2006-2010$ & x & \\
Costa Rica & 56 & 57 & 57 & 100 & $2006-2010$ & x & \\
El Salvador & 58 & 84 & 72 & 85,0 & $2006-2009$ & x & \\
Guatemala & 68 & 158 & 97 & 61,4 & $2008-2012$ & x & \\
Honduras & 57 & 128 & 91 & 71,1 & $2006-2010$ & x & \\
México & 63 & 500 & 128 & 25,6 & $2006-2009$ & x & \\
Panamá & 71 & 71 & 64 & 90,0 & $2009-2013$ & x & x \\
Paraguay & 69 & 80 & 72 & 90,0 & $2008-2013$ & x & \\
Perú & 61 & 120 & 96 & 80,0 & $2006-2011$ & x & \\
Colombia-b & 83 & 165 & 91 & 55,0 & $2010-2014$ & x & x \\
Costa Rica-b & 78 & 57 & 56 & 98,3 & $2010-2014$ & x & x \\
El Salvador-b & 70 & 84 & 68 & 81,0 & $2009-2011$ & x & x \\
Honduras-b & 74 & 128 & 91 & 70,0 & $2010-2014$ & x & x \\
México-b & 79 & 500 & 98 & 19,6 & $2009-2011$ & x & x \\
Perú-b & 84 & 130 & 93 & 72,0 & $2011-2016$ & x & x \\
\hline (n) & & & 1.379 & & & 1.379 & 561 \\
\hline
\end{tabular}

Nota: Se puede consultar información adicional sobre el proyecto, acceder a las bases de datos y los cuestionarios de estos y otros estudios en: http://americo.usal.es/oir/elites/index.htm 


\section{Descripción de las variables incluidas en los análisis y codificación}

\begin{tabular}{ll}
\hline \multicolumn{1}{c}{ Variables } & \multicolumn{1}{c}{ Categorías } \\
\hline Educación & $\begin{array}{l}\text { Nivel medio (inferior a grado); Nivel alto } \\
\text { (grado y postgrado) }\end{array}$ \\
Experiencia legislativa & Novato; Dos legislaturas o más \\
Cargo de elección popular & Sí; No \\
Cargo de designación & Sí; No \\
Cargo de partido & Sí; No \\
Índice de Desarrollo Humano (IDH) & 0 Bajo- 100 Alto \\
Ingresos & 1 entre 1.000 y 4.000 dólares; 2 entre 4.001 y 7.000 \\
dólares; 3 entre 7.001 y 10.000 dólares; 4 más de 10.001 \\
dólares \\
Familiares en política & Hombre; Mujer \\
Partido & Sí; No \\
Edad & De gobierno; De oposición \\
Ideología & Número de años \\
& 1 izquierda-10 derecha
\end{tabular}

María del Mar Martínez Rosón es becaria postdoctoral en la Universidad Autónoma de Barcelona (España). Es doctora en Ciencia Política por la Universidad de Salamanca (España) y Máster en Estudios latinoamericanos por la misma Universidad. Ha sido becaria posdoctoral en la Universidad de Tulane en Nueva Orleans (EE.UU.) y ha realizado estancias de investigación en la Universidad de Michigan (EE.UU.), la Universidad de Boston (EE.UU.) y la Universidad de Notre Dame (EE.UU.). E-mail: mar.martinez@uab.es 